Dieses Dokument ist eine Zweitveröffentlichung von:

Tim Ziemer u. Rolf Bader

Complex point source model to calculate the sound field radiated from musical instruments

Veröffentlicht in:

Proceedings of Meetings on Acoustics, Volume 25, Issue 1

Verlag: AIP Publishing | Jahr: 2015

DOI: https://doi.org/10.1121/2.0000122

Es gelten die Regelungen des Urheberrechts, https://rightsstatements.org/page/lnC/1.0/?language $=$ de

Bereitgestellt von:

musiconn.publish - https://musiconn.qucosa.de 


\section{Complex point source model to calculate the sound field radiated from musical instruments}

Tim Ziemer, and Rolf Bader

Citation: Proc. Mtgs. Acoust. 25, 035001 (2015); doi: 10.1121/2.0000122

View online: https://doi.org/10.1121/2.0000122

View Table of Contents: https://asa.scitation.org/toc/pma/25/1

Published by the Acoustical Society of America

\section{ARTICLES YOU MAY BE INTERESTED IN}

Psychoacoustic sonification design for navigation in surgical interventions

Proceedings of Meetings on Acoustics 30, 050005 (2017); https://doi.org/10.1121/2.0000557

Perceptual sound field synthesis concept for music presentation

Proceedings of Meetings on Acoustics 30, 015016 (2017); https://doi.org/10.1121/2.0000661

Perceptual evaluation of violin radiation characteristics in a wave field synthesis system

Proceedings of Meetings on Acoustics 30, 035001 (2017); https://doi.org/10.1121/2.0000524

Complex point source model to calculate the sound field radiated from musical instruments

The Journal of the Acoustical Society of America 138, 1936 (2015); https://doi.org/10.1121/1.4934107

Perceptually motivated sound field synthesis for music presentation

The Journal of the Acoustical Society of America 141, 3997 (2017); https://doi.org/10.1121/1.4989162

Simulating the lateral line with low-frequency nearfield acoustic holography based on a vector hydrophone array for short-range navigation in littoral waters

The Journal of the Acoustical Society of America 138, 1897 (2015); https://doi.org/10.1121/1.4933959

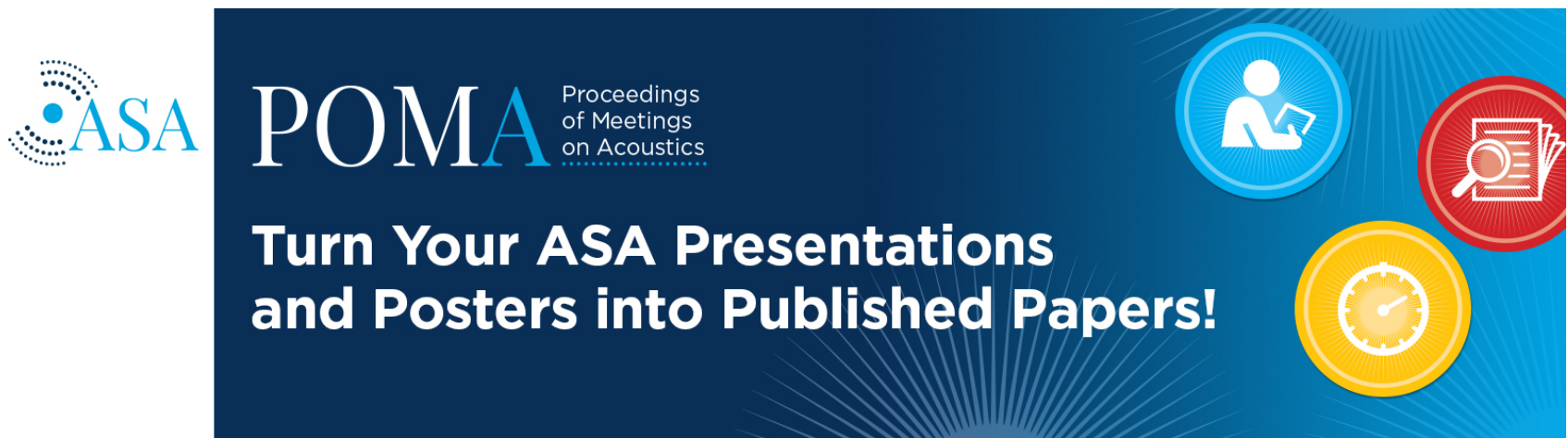




\section{Proceedings of Meetings on Acoustics}

Volume 25

http://acousticalsociety.org/

\section{0th Meeting of the Acoustical Society of America}

Jacksonville, Florida

02-06 November 2015

\section{Musical Acoustics: Paper 5aMU7}

\section{Complex point source model to calculate the sound field radiated from musical instruments}

Tim Ziemer and Rolf Bader

Institute of Systematic Musicology, University of Hamburg, Germany.; tim.ziemer@uni-hamburg.de; r_bader@t-online.de

A simple method is described to record the radiated sound of musical instruments and to extrapolate the sound field to distances further away from the source. This is achieved by considering instruments as complex point sources. It is demonstrated that this simplification method yields plausible results not only for small instruments like the shakuhachi but also for larger instruments such as the double bass: The amplitude decays in a given manner and calculated interaural signal differences reaching the listener decrease with increasing distance to the source. The method can be applied to analyze the sound radiation characteristics as well as the radiated sound field in a listening region regardless of room acoustical influences. Implementations in terms of room acoustical simulations, spatial additive synthesis and sound field synthesis are discussed. 


\section{INTRODUCTION}

The sound radiation characteristics of musical instruments have been investigated by means of microphone array measurements by many authors. Especially studies by Meyer and Pätynen and Lokki reveal comprehensive insights in the radiation characteristics of symphony orchestra instruments [1,2]. Typically, amplitudes measured in the far field of instruments are expressed as a function of angle. This approach already implies that the sound source is considered to be a point in the origin of a polar coordinate system having a complex radiation pattern. This simplification is also known as multipole point source or higher mode radiator in room acoustical planning [3] and is applied as multipole expansion or single point multipole method in the field of sound sources reconstruction by means of acoustic holography $[4,5]$.

In the field of subjective room acoustics researchers like Beranek have found objective parameters that correlate with subjective impressions [6,7]. Particularly the binaural quality index is reported to correlate with the impression of apparent source width. Therefore, its applicability for the investigation of pure direct sound is discussed.

This paper has three main sections. In the first section the method is described to measure, extrapolate and analyze sound radiation, including measurement setup, description of the complex point source model and proposition of quantitative analysis. In the second section exemplary analysis results are presented for a double bass and a shakuhachi. The method, its limitations and the applicability of the proposed measures are discussed in the final section including prospects of further investigations, developments and potential implementations by means of spatial additive synthesis or sound field synthesis.

\section{METHOD}

The used measurement setup will be described next. Then the idea of the complex point source model is described and it is explained how to extrapolate the recorded sound away from the source. At last some parameters are proposed which describe the radiation characteristics as well as the radiated sound at several receiver distances.

\subsection{Measurement Setup}

A circular microphone array consisting of 128 omnidirectional electret microphones with a frequency range from $20 \mathrm{~Hz}$ to $20 \mathrm{kHz}$ is hung up horizontally in a free field room. It has a radius of $1 \mathrm{~m}$, so the array has one microphone every $2.8^{\circ}$ or every $0.05 \mathrm{~m}$. An instrumentalist is placed with his head in the center of the microphone array facing microphone number 1 . The instrumentalist plays a single note without remarkable articulation, tremolo, crescendo or vibrato. No shaker is used to drive the instrument as only the instrumentalist creates natural wave shadow. It is recorded simultaneously and digitalized with a sample rate of $48 \mathrm{kHz}$ and a sample depth of 24 bit, yielding $M=128$ time series

$$
p\left(t, \mathbf{r}_{m}\right)
$$

where $\mathbf{r}_{m}$ is the position vector of the $m$ th microphone in polar coordinates consisting of the horizontal angle $\varphi$ and the distance $r$ :

$$
\mathbf{r}_{m}=\left[\begin{array}{c}
1 \mathrm{~m} \\
\frac{2 \pi m}{128}
\end{array}\right]
$$

Several notes are played to cover the whole frequency area. However, single notes are analyzed and discussed throughout this paper. 


\subsection{The Sum Spectrum}

From a spectrum all radiated partials can be identified. It is not sufficient, however, to calculate the spectrum from one microphone recording only. Those frequencies that are poorly radiated to that specific microphone position could be overseen. To find all radiated partials reliably, it is meaningful to calculate one sum spectrum from all recordings. A discrete Fourier transform (DFT) over one second of quasi-stationary sound for each microphone recording yields $M=128$ spectra

$$
P\left(\omega, \mathbf{r}_{m}\right)
$$

consisting of 24000 complex amplitudes below the temporal aliasing frequency. These consist of a real part $\Re\left[P\left(\omega, \mathbf{r}_{m}\right)\right]$ and an imaginary part $\Im\left[P\left(\omega, \mathbf{r}_{m}\right)\right]$. However, more interesting are the amplitude

$$
\hat{A}\left(\omega, \mathbf{r}_{m}\right)=\sqrt{\Re^{2}\left[P\left(\omega, \mathbf{r}_{m}\right)\right]+\Im^{2}\left[P\left(\omega, \mathbf{r}_{m}\right)\right]}
$$

and the phase

$$
\phi\left(\omega, \mathbf{r}_{m}\right)=\arctan \left(\frac{\Im\left[P\left(\omega, \mathbf{r}_{m}\right)\right]}{\Re\left[P\left(\omega, \mathbf{r}_{m}\right)\right]}\right) .
$$

In this paper the phase is considered as ranging from 0 to $2 \pi$. By simply adding up the amplitudes from all recordings, neglecting phase information, one sum spectrum

$$
\hat{A}(\omega, 1 \mathrm{~m})=\sum_{m=1}^{M} \hat{A}\left(\omega, 1 \mathrm{~m}, \varphi_{m}\right)
$$

can be calculated. It contains the sum over all angles at the recording distance $r=1 \mathrm{~m}$. From this sum spectrum all partials can be identified and analyzed individually. Some authors prefer to combine the individual radiation patterns within a certain frequency band-like octave bands or critical bands-to one common radiation pattern of that frequency region $[1,8]$. The advantage of this approach is that it gives global results of how instruments tend to radiate certain frequency regions. However, this method might average out the actual and often very complicated radiation patterns of single frequencies. Therefore, the proposed method aims at analyzing all partials individually before summarizing them to more global values.

\subsection{The Complex Point Source Model}

In two dimensions the spatial propagation of a spectrum from a point source at the origin $P_{\mathbf{Q}}(\omega, 0)$ in a free field is described by the inhomogeneous Helmholtz equation in polar coordinates. By separation of variables it can be written as one equation for the distance $r$

$$
\begin{aligned}
& \frac{1}{r^{2}} \frac{\mathrm{d}\left(r^{2} \frac{\mathrm{d} G(\omega, r)}{\mathrm{d} r}\right)}{\mathrm{d} r} \\
& +k^{2} G(\omega, r)-\frac{n(n+1)}{r^{2}} G(\omega, r)=\delta\left(r-r_{0}\right),
\end{aligned}
$$

and one for the azimuth angle $\varphi$

$$
\frac{\mathrm{d}^{2} \Gamma(\omega, \varphi)}{\mathrm{d} \varphi^{2}}+m^{2} \Gamma(\omega, \varphi)=0,
$$

where $\delta\left(r-r_{0}\right)$ is the Dirac delta function and $m$ and $n$ are integers [4]. A valid solution for distance part is the well-known free field Green's function

$$
G(\omega, r)=\frac{e^{-\imath k r}}{r}
$$


where $\imath$ is the imaginary unit. It describes the propagation of the source spectrum to any further distance. As $G(\omega, r)$ is assumed to be equal for all point sources, $\Gamma(\omega, \varphi)$ fully describes the sound radiation characteristic of the source in two dimensions. In the literature, it is sometimes referred to as far field signature function or far field directivity pattern $[4,9]$. In case of a circular microphone array arranged around a complex point source in its center the far field signature function is implicitly measured by the 128 recordings since

$$
P\left(\omega, 1 \mathrm{~m}, \varphi_{m}\right)=P_{\mathbf{Q}}(\omega, 0) G(\omega, 1 \mathrm{~m}) \Gamma\left(\omega, \varphi_{m}\right) .
$$

(10) sates that the recorded spectra $P\left(\omega, \mathbf{r}_{m}\right)$ are nothing but the original source spectrum at the origin $P_{\mathbf{Q}}(\omega, 0)$ propagated through the free field according to the given distance function $G(\omega, 1 \mathrm{~m})=\frac{e^{-\imath k 1}}{1}$, amplified by the complex angular factor $\Gamma\left(\omega, \varphi_{m}\right)$ for the specific angle between the origin and the $m$ th microphone. In other words-because the source spectrum and the distance function are independent of the angle - the recorded spectra are directly proportional to the far field signature function of the complex point source, only reduced in amplitude and shifted in phase. As we have discrete microphone positions, we practically sample $\Gamma(\omega, \varphi)$. To receive the radiated spectra at other angles, we could either interpolate or decompose the measured $\Gamma$ to a finite set of circular harmonics. To calculate the spectra at other distances $r_{x}$, the recorded spectra only have to be divided by the distance function for $1 \mathrm{~m}$ and then multiplied by a distance function for the according distance:

$$
P\left(\omega, r_{x}, \varphi_{m}\right)=\frac{P\left(\omega, 1 \mathrm{~m}, \varphi_{m}\right)}{G(\omega, 1 \mathrm{~m})} G\left(\omega, r_{x}\right)
$$

So wandering along the extended connection line between source and observer in the far field does not change the spectrum much except for an amplitude decrease and a relative phase change which are the same for all directions and only dependent on frequency and distance. Thus, the spectrum measured at a microphone recording position represents the spectrum not only for this very position but for every position in the far field having the same angle to the source.

Most symphony orchestra instruments radiate lower frequencies nearly as monopoles, anyway [1,2]. This means, although the microphones may lie in the near field for low frequencies, the recorded $\Gamma$ should at least resemble the far field signature function since a monopole has no deviant near field effects. Furthermore, as the microphones are omnidirectional pressure receivers, no proximity effect should arise. Thus, low frequencies should not be overemphasized compared to higher partials.

With the described setup, applying the complex point source model, an infinite number of parameters could be gained from actual measurements as well as from the extrapolated sound field. Some parameters that seem to be suitable to describe the radiation characteristics of musical instruments as well as the sound that actually reaches a listener at different listening positions are proposed in the following.

\subsection{Physical Measures}

From the amplitudes (4) and phases (5) interaural level and phase differences $I L D(\omega, \mathbf{r})$ and $I P D(\omega, \mathbf{r})$ can be calculated for listeners positioned at 128 angles at a distance of $1 \mathrm{~m}$ :

$$
\operatorname{ILD}(\omega, \mathbf{r})=20 \lg \left(\frac{\hat{A}\left(\omega, 1 \mathrm{~m}, \varphi_{L}\right)}{\hat{A}\left(\omega, 1 \mathrm{~m}, \varphi_{R}\right)}\right)
$$

and

$$
\operatorname{IPD}(\omega, \mathbf{r})=\left|\phi\left(\omega, 1 \mathrm{~m}, \varphi_{L}\right)-\phi\left(\omega, 1 \mathrm{~m}, \varphi_{R}\right)\right| .
$$

By extrapolating (4) and (5) via (11) ILD and IPD of further distance can be calculated accordingly. Here, the subscripts $L$ and $R$ denote "left" and "right" ear, the $I P D$ is defined as lying between 0 and $\pi$. Note 
that no actual listener is present. Instead, microphone positions with the distance of two ears (about 0.15 $\mathrm{m})$ are taken as left and right ear: This means at $1 \mathrm{~m}$ every third microphone signal, at $1.5 \mathrm{~m}$ every second extrapolated microphone signal, and at $3 \mathrm{~m}$ all extrapolated signals of adjacent microphones are compared. Other listening distances are neglected as they would require manipulations in terms of interpolation or (de)-composition, see section 2.3. No head related transfer function (HRTF) is implemented. As the source is assumed to be a single point right in front of a listener, the HRTF would have no binaural effect. Only a spectral filtering would result which is quite individual. A generic or personal HRTF could be implemented later on. However, the term "interaural" is kept throughout this paper as measured signal differences without the physical presence of an actual listener are assumed to be equivalent to interaural differences.

Another interaural parameter known from room acoustical investigations is the binaural quality index $(B Q I)$. It is related to the perception of apparent source width (ASW). The $B Q I$ is derived from the early interaural cross correlation coefficient $I A C C_{E}$ which is the maximum absolute value of the early inter aural cross correlation coefficient function $I A C F_{E}$ as calculated from dummy head recordings of the $500 \mathrm{~Hz}$ to the $2 \mathrm{kHz}$ octave band:

$$
\begin{gathered}
I A C F_{E}(\tau, \mathbf{r})=\frac{\int_{0}^{80 \mathrm{~ms}} p_{L}(t) p_{R}(t+\tau) \mathrm{d} t}{\sqrt{\int_{0}^{80 \mathrm{~ms}} p_{L}^{2}(t) \mathrm{d} t \int_{0}^{80 \mathrm{~ms}} p_{R}^{2}(t) \mathrm{d} t}} \\
I A C C_{E}(\mathbf{r})=\max |I A C F(\tau, \mathbf{r})| \\
B Q I_{E}(\mathbf{r})=1-I A C C
\end{gathered}
$$

Note that this parameter is gained from the temporal signals, not the spectrum. The subscript $E$ denotes an integral over the first $80 \mathrm{~ms}$ point out that especially the direct sound and early reflections are of importance for the ASW. In room acoustical investigations $\tau= \pm 1 \mathrm{~ms}$ accounts for the fact that a laterally arriving signal creates an interaural time difference of up to $1 \mathrm{~ms}$. Still, a lateral sound source does not sound wider than a frontal source, so the influence of this time difference is eliminated by $\tau$. However, as the source position is assumed to be always frontal in this setup, a $B Q I_{\text {dir }}(\mathbf{r})$ with $\tau=0$ is more meaningful for pure direct sound and can be expected to correlate with the impression of ASW.

From everyday experience as well as from room acoustical investigations we qualitatively know how the radiated sound of a musical instrument behaves. With increasing distance the amplitude gets lower and the source sounds smaller as interaural differences decrease. What we expect to observe in the described measures is listed in the following:

- $\hat{A}$ decay with distance

- $\phi$ shift with distance

- $I L D$ decrease with distance

- IPD decrease with distance

- $B Q I$ decrease with distance

- $\hat{A}$ of low frequencies tends to be omnidirectional

- Patterns of $\hat{A}$ and $\phi$ are more complicated for higher frequencies

- Patterns of $\hat{A}$ and $\phi$ are more complex for complicated geometries

These expectations should be met even when applying the complex point source model. 


\section{Results}

A sum spectrum of a stringed double bass note is illustrated in fig. 1 (a). Compared to the spectrum of one single microphone recording only, no partial can be overseen. $N=155$ partials are present in the octave bands from $63 \mathrm{~Hz}$ to $8 \mathrm{kHz}$.

As mentioned earlier, the far field signature function consists of an amplitude and a phase for each frequency at each angle, given by (4) and (5). The amplitude per angle of a low and a high double bass frequency as well as a high shakuhachi partial are plotted for 128 angles and 3 distances in fig. 1 . The plots show the expected typical features partly reported already by [1,2]: It can be seen that the low double bass frequency (b) radiates directionally uniform, having a mean amplitude of $118.77 \mathrm{~dB}$ referred to the lowest measured amplitude of all partials at a distance of $1 \mathrm{~m}$. The higher frequency (c) is softer and has a more complicated radiation pattern. Compared to that, the same frequency radiated from a shakuhachi (d) looks much more symmetric. Note that the amplitudes at the other distances are directly proportional to those at a distance of $1 \mathrm{~m}$ as they are calculated by (9). The value in the brackets is the actual frequency $f=\frac{\omega}{2 \pi}$ not the angular frequency $\omega$.

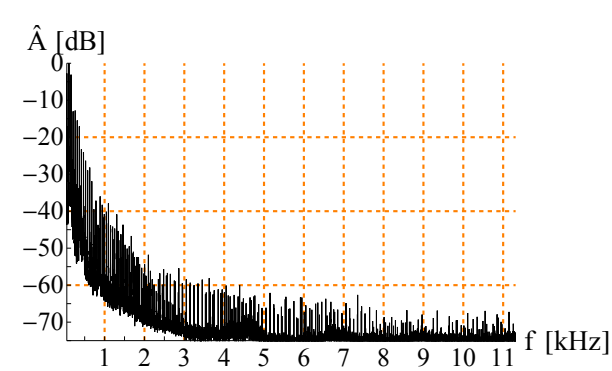

(a) Sum Spectrum (Double Bass)

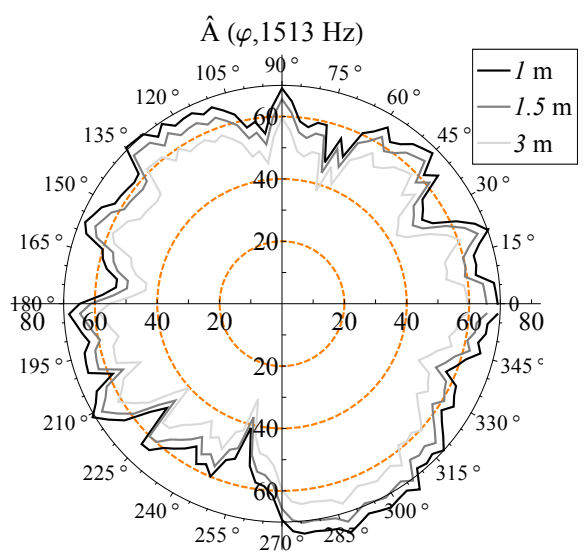

(c) $\hat{A}$ Double Bass (high)

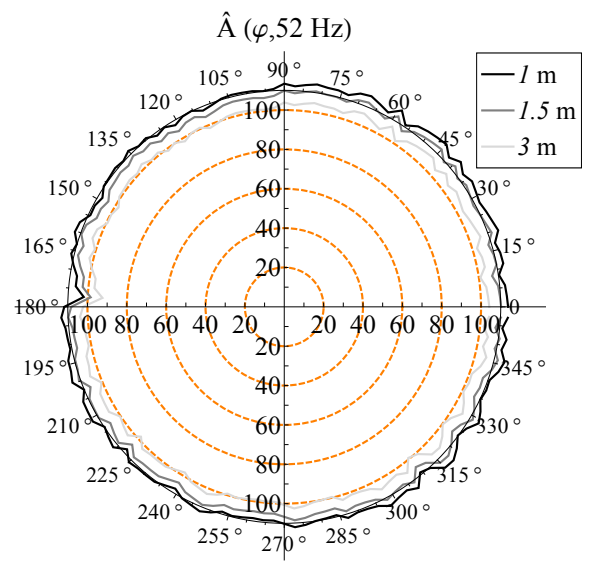

(b) $\hat{A}$ Double Bass (low)

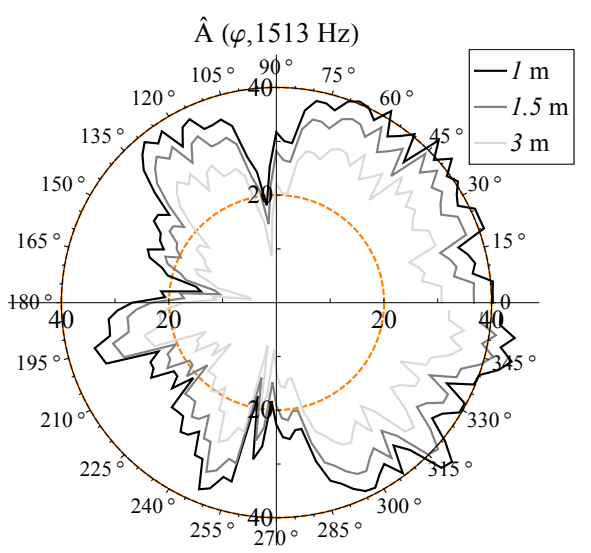

(d) $\hat{A}$ Shakuhachi (high)

Figure 1: Sum spectrum of a double bass (a) and amplitudes of a low (b) and a high (c) double bass and a high shakuhachi (d) frequency. The double bass has $N=155$ partials from the $63 \mathrm{~Hz}$ to the $8 \mathrm{kHz}$ octave band. Its low frequency radiation resembles a monopole, the radiation of the high frequency is more complicated, the shakuhachi pattern is more complicated than the low frequency but more symmetric than the same frequency of the double bass. 
As can be seen in fig. 2, plotting the phase shows a dipole character of the low double bass frequency (a). Two regions of rather constant phase are visible having a difference of about $\pi$. The high double bass frequency (b) reveals much more diverse phases. The shakuhachi (c) radiates the same frequency with much more steady phase relations.

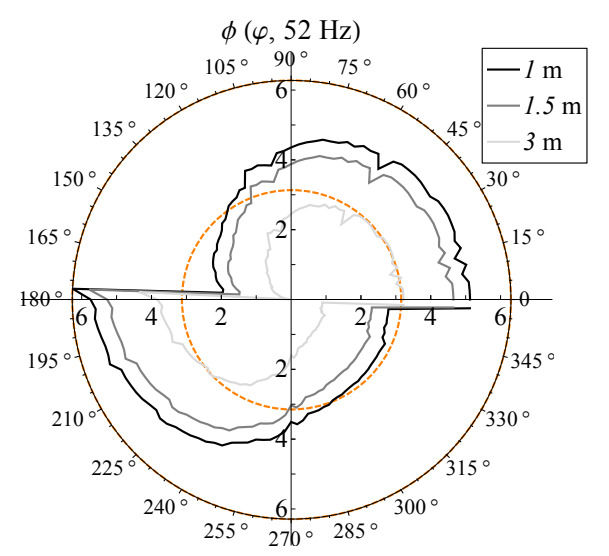

(a) $\phi$ Double Bass (low)

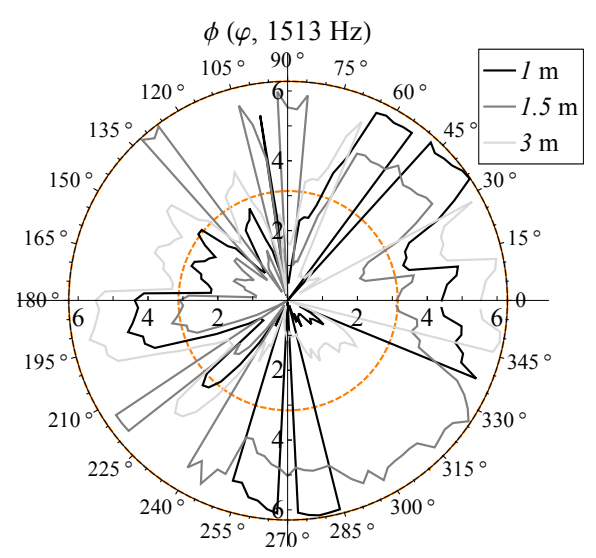

(b) $\phi$ Double Bass (high)

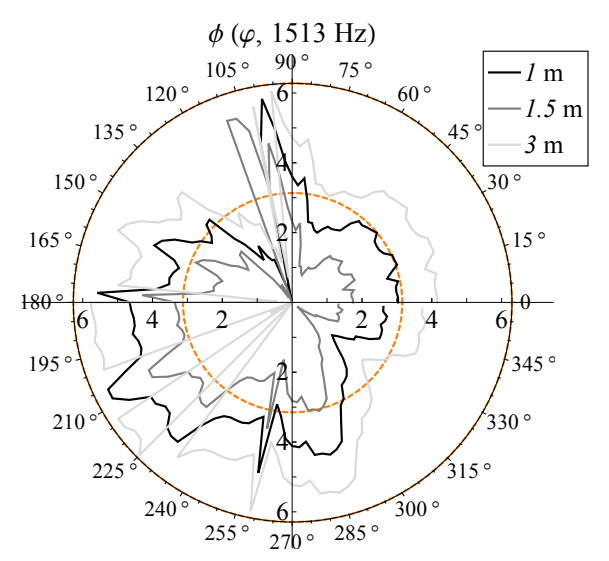

(c) $\phi$ Shakuhachi (high)

Figure 2: Phase of a low (a) and a high (b) double bass and a high shakuhachi (c) frequency. For the low double pass frequency a dipole character is clearly visible. The phase pattern of the high partial has many fluctuations. Compared to that the high frequency phase pattern of the shakuhachi is more smooth.

The $B Q I(\mathbf{r})$ of a quasi stationary double bass sound is plotted in fig. 3 (a). It is much lower than values in room acoustical investigations and decreases with increasing distance from 0.038 over 0.024 to 0.011 . Mean $I L D$ for the double bass are also plotted in fig. 3. ILD per frequency (b) increase until about 1.5 $\mathrm{kHz}$ and stay rather constant for higher frequencies except for sporadic outliers. Also, $I L D$ of individual frequencies tend to decrease with distance. Overall, mean $I L D$ of all frequencies (c) tend to decrease with distance at most angles, to average from $5.98 \mathrm{~dB}$ over $5.37 \mathrm{~dB}$ to $4.53 \mathrm{~dB}$. Likewise, IPD decrease with distance from 0.7 over 0.61 to 0.48 (d). Mean $I L D, I P D$ and $B Q I$ of double bass and shakuhachi are summarized in tab. 1 for the three distances. They all behave as expected: $I L D$ of the low double bass frequency are smaller than of the high shakuhachi frequency which are again smaller than for the high double bass frequency. This is also true for $I P D$. They all decrease with distance. Overall, the double bass has larger interaural differences than the shakuhachi. 


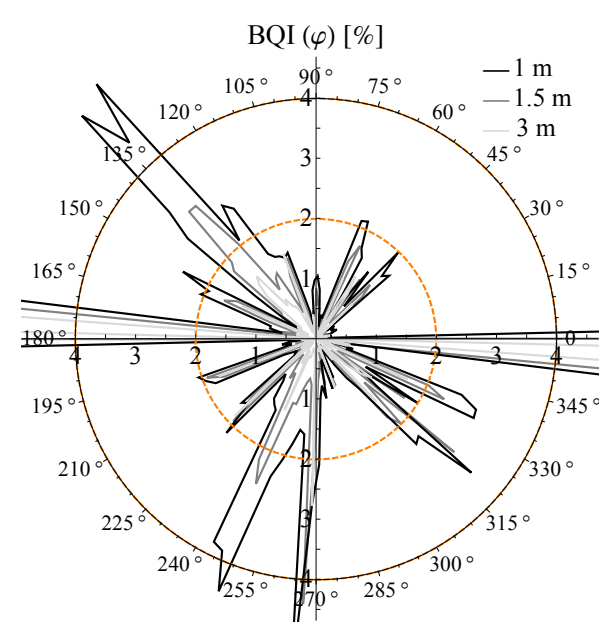

(a) BQI Double Bass

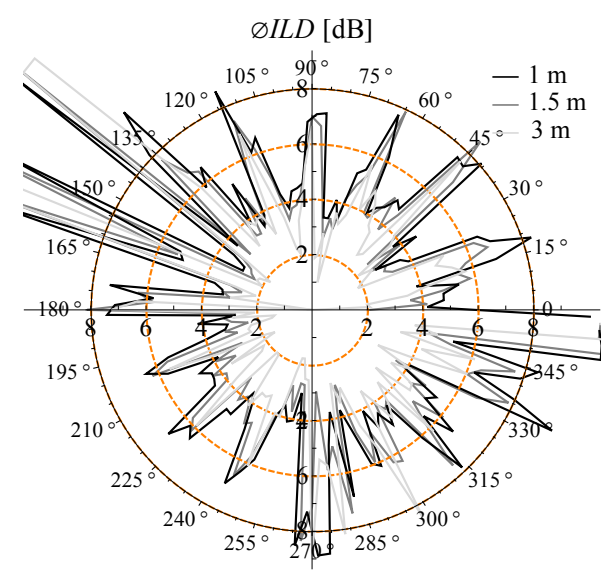

(c) $\oslash I L D(\varphi, r)$ Double Bass

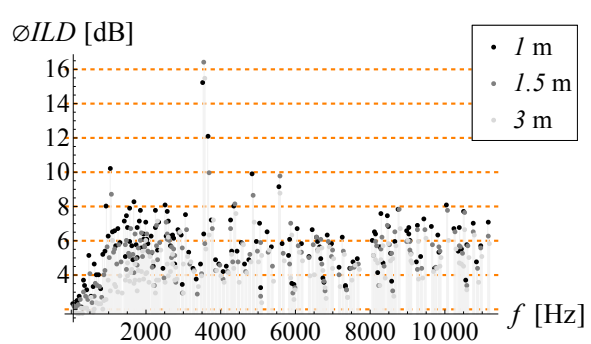

(b) $\oslash I L D(\omega, r)$ Double Bass

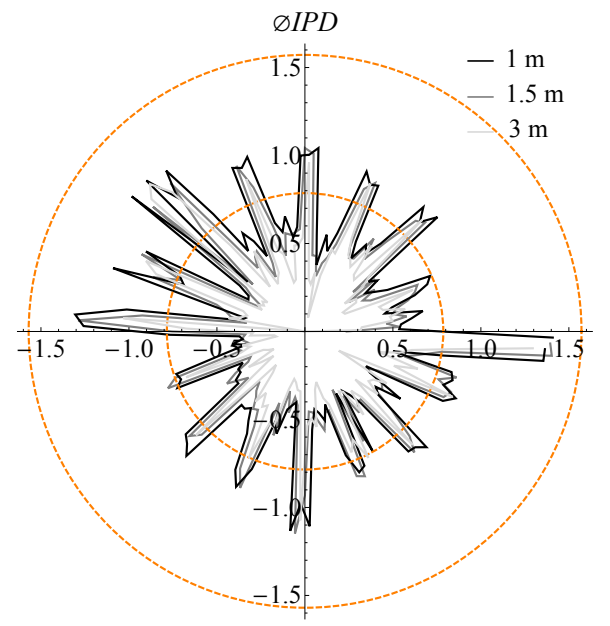

(d) $\oslash I P D(\varphi, r)$ Double Bass

Figure 3: $B Q I$ (a), mean $I L D$ over all angles (b) and all frequencies (c) and $I P D$ over all frequencies (d). $B Q I, I L D$ and $I P D$ of most frequencies and angles decrease over distance.

\begin{tabular}{|l||l|l|l|}
\hline value & $1 \mathrm{~m}$ & $1.5 \mathrm{~m}$ & $3 \mathrm{~m}$ \\
\hline$\oslash I L D_{\mathrm{l}, \mathrm{db}}$ & $2.14 \mathrm{~dB}$ & $2.02 . \mathrm{dB}$ & $1.73 \mathrm{~dB}$ \\
$\oslash I L D_{\mathrm{h}, \mathrm{db}}$ & $5.42 \mathrm{~dB}$ & $4.31 \mathrm{~dB}$ & $3.1 \mathrm{~dB}$ \\
$\oslash I L D_{\mathrm{db}}$ & $5.98 \mathrm{~dB}$ & $5.37 \mathrm{~dB}$ & $4.53 \mathrm{~dB}$ \\
$\oslash I L D_{\mathrm{h}, \mathrm{sh}}$ & $4.11 \mathrm{~dB}$ & $3.62 \mathrm{~dB}$ & $2.64 \mathrm{~dB}$ \\
$\oslash I L D_{\mathrm{sh}}$ & $4.29 \mathrm{~dB}$ & $3.6 \mathrm{~dB}$ & $3.12 \mathrm{~dB}$ \\
\hline$\oslash I P D_{\mathrm{l}, \mathrm{db}}$ & 0.23 & 0.16 & 0.1 \\
$\oslash I P D_{\mathrm{h}, \mathrm{db}}$ & 0.99 & 0.71 & 0.4 \\
$\oslash I P D_{\mathrm{db}}$ & 0.7 & 0.61 & 0.48 \\
$\oslash I P D_{\mathrm{h}, \mathrm{sh}}$ & 0.55 & 0.45 & 0.36 \\
$\oslash I P D_{\mathrm{sh}}$ & 0.55 & 0.44 & 0.26 \\
\hline$\oslash B Q I_{\mathrm{db}}$ & 0.038 & 0.024 & 0.011 \\
$\oslash B Q I_{\mathrm{sh}}$ & 0.022 & 0.013 & 0.007 \\
\hline
\end{tabular}

Table 1: Mean interaural differences of a single low (l) and high (h) double bass (db) and shakuhachi (sh) frequency and of all frequencies. Higher notes create larger interaural differences, double bass even more than shakuhachi. All interaural differences decrease with distance. 


\subsection{Discussion}

A method has been introduced to measure the direct sound of musical instruments by means of a microphone array and to simplify the the instrument as complex point source. This simplification yields complex amplitudes for several discrete angles showing the well known radiation properties of musical instruments. In addition, the simplification can be applied to extrapolate the sound field to different listening positions. From these extrapolated sound fields $I L D(\omega, \mathbf{r}), I P D(\omega, \mathbf{r})$ and $B Q I(\mathbf{r})$ as perceived by a listener can be calculated for several listening angles and distances. These seem to be proper measures to investigate the relationship between the radiation and the perception of source extent or distance, as they behave in a natural way. Despite the drastic physical simplification, the model satisfies all expectations listed above: Amplitude decays and phase shifts according to the chosen transfer function. Furthermore, all calculated interaural differences are larger for the double bass than for the shakuhachi and decrease with increasing distance. Low frequencies show more omnidirectional radiations than higher frequencies and the radiation pattern of a high double bass frequency is more complicated than the same frequency radiated from a shakuhachi especially concerning phase. The chosen radius of $1 \mathrm{~m}$ is just the right distance, lying in the near field only for exactly those frequencies whose radiation characteristic tends to be omnidirectional and very similar in the near and the far field. For higher frequencies the distance can be considered as far field being several wave lengths away from the source. It is conspicuous, however, the fundamental is only $2.73 \mathrm{~dB}$ weaker than the first overtone which contradicts observations by e.g. Askenfelt [10] who observed values between 12 and $20 \mathrm{~dB}$. One reason for that may certainly be that the measurement room cannot be considered anechoic in this frequency region. Still, the development of calculated interaural differences remains plausible so the model seems suitable even for such large instruments and low frequencies. Note however, that for several types of instruments different playing techniques, different strings or fingerings may result in different radiation patterns even for the same frequency $[1,2,11]$.

Fifteen instruments have been recorded with the described setup. Synthesizing the radiation characteristics of musical instruments is a research topic of growing interest [9]. The presented way of measuring and extrapolating the sound radiation of musical instruments has already been implemented in loudspeaker systems in terms of spatial additive synthesis and sound field synthesis [12-14] resulting in the perception of increased spaciousness compared to mono and stereo setups. Corteel reports an increased perceived "presence" of virtual sources synthesized with a more complex radiation pattern than a monopole [15]. Baalman created complex radiation patterns by a distribution of monopole sources in a wave field synthesis application and discovered an improved perceived naturalness [16]. Jacques et al. report that listeners were able to hear the orientation but had problems localizing a source whose radiation characteristic has been resynthesized by means of virtual super cardioid sources in a wave field synthesis setup [17]. Otondo and Rindel proposed a method similar to complex point sources with up to 5 azimuth and 5 polar angle factors for three-dimensional implementation in ray-tracing softwares [18]. Auralizations provided an improved naturalness of timbre but inconsistent judgments concerning spaciousness.

Analyzing more instruments in the presented way and synthesizing their radiation patterns will make it possible to directly investigate the relationship between the physical radiation characteristics and the psychological perception of apparent source width by keeping all other parameters constant. One can think of several other monaural and binaural parameters, e.g. derived from the field of subjective room acoustics, to quantify the relationship between the physics of a sound field and the perception of spaciousness. The relationship between the actual physical source width in degrees and the sound field measured and extrapolated to several listening positions by means of the complex point source method has already been investigated for 8 instruments at 3 distances in [19]. Besides research, there are of course also potential application areas in the entertainment sector, like audio systems for music and film.8 


\section{Conclusion and Prospects}

Measuring the sound radiation of musical instruments is always a compromise between physical accuracy and computational demands. The strength of the presented complex point source model is that it is a simplification which makes it easy to measure, analyze and compare the sound radiation characteristics of musical instruments. Extrapolation of the sound to positions beyond the microphone positions is rather simple. Though physically untrue, considering musical instruments as complex point sources yields plausible results even for instruments as large as a double bass. It has to be investigated, however, a) if the model is even applicable for larger instruments such as a harpsichord and b) how much the extrapolated sound field resemble the actual sound field. A method with the opposite strength would be to backpropagate the radiated sound to points on the instrumental body and air holes by means of nearfield acoustic holography as in [20]. Then, the sound field can be forward propagated by superposition of point sources for every angle and distance within a half space [21]. Such a method is close to the actual physics but requires high computational costs.

\section{References}

[1] Jürgen Meyer, Acoustics and the Performance of Music. Manual for Acousticians, Audio Engineers, Musicians, Architects and Musical Instrument Makers, Springer, Bergkirchen, 5th ed. edition, 2009.

[2] Jukka Pätynen and Tapio Lokki, "Directivities of symphony orchestra instruments," Acta Acustica United With Acustica, vol. 96, pp. 138-167, 2010.

[3] Fridolin Mechel, Room Acoustical Fields, Springer, Berlin, Heidelberg, 2013.

[4] Earl G. Williams, Fourier Acoustics. Sound Radiation and Nearfield Acoustical Holography, Academic Press, Cambridge, 1999.

[5] Marcelo Bruno S. Magalhães and Roberto A. Tenenbaum, "Sound sources reconstruction techniques: A review of their evolution and new trends," Acta Acustica United With Acustica, vol. 90, pp. 199-220, 2004.

[6] Leo Leroy Beranek, Concert Halls and Opera Houses: Music, Acoustics, and Architecture, New York, 2 edition, 2004.

[7] Leo Leroy Beranek, "Subjective rank-orderings and acoustical measurements for fifty-eight concert halls," Acta Acustica United With Acustica, vol. 89, pp. 494-508, 2003.

[8] Felipe Otondo and Jens Holger Rindel, "The influence of the directivity of musical iinstrument in a room," Acta Acustica United With Acustica, vol. 90, pp. 1178-1184, 2004.

[9] Jens Ahrens, Analytic Methods of Sound Field Synthesis, Springer, Berlin, Heidelberg, 2012.

[10] Anders Askenfelt, The Science of String Instruments, chapter Double Bass, pp. 259-277, Springer, 2010.

[11] Fabian Hohl and Franz Zotter, "Similarity of musical instrument radiation-patterns in pitch and partial," in Fortschritte der Akustik, DAGA, Berlin, 2010.

[12] Tim Ziemer, "Wave field synthesis by an octupole speaker system," SysMus09 Proceedings, pp. 89-93, 112009. 
[13] Tim Ziemer, "A psychoacoustic approach to wave field synthesis," in Audio Engineering Society Conference: 42nd International Conference: Semantic Audio, 7 2011, pp. 191-197.

[14] Tim Ziemer and Rolf Bader, "Implementing the radiation characteristics of musical instruments in a psychoacoustic spund field synthesis system," in Audio Engineering Society Convention 139, New York, 2015.

[15] Etienne Corteel, "Synthesis of directional sources using wave field synthesis, possibilities, and limitations," EURASIP Journal on Advances in Signal Processing, vol. 2007, 2007.

[16] Marije Baalman, On Wave Field Synthesis and electro-acoustic music, with a particular focus on the reproduction of arbitrarily shaped sound sources, VDM, Saarbrücken, 2008.

[17] Roland Jacques, Bernhard Albrecht, Frank Melchior, and Diemer de Vries, "An approach for multichannel recording and reproduction of a sound source directivity," in Audio Engineering Society Convention 119, New York, 102005.

[18] Felipe Otondo and Jens Holger Rindel, "A new method for the readiation representation of musical iinstrument in auralization," Acta Acustica United With Acustica, vol. 91, pp. 902-906, 2005.

[19] Tim Ziemer, "Adapting room acoustic parameters to explain apparent source width of direct sound," in 'Musik und Wohlbefinden'. 31. Jahrestagung der DGM, Oldenburg, 2015.

[20] Rolf Bader, "Reconstruction of radiating sound fields using minimum energy method," J. Acoust. Soc. Am., vol. 127, no. 1, pp. 300-308, 2010.

[21] Rolf Bader, "Microphone array," in Handbook of Acoustics, Thomas D. Rossing, Ed. Springer, Berlin Heidelberg, 2014. 